\title{
A 10-year retrospective study of alterative aeroallergens sensitization spectrum in urban children with allergic rhinitis
}

This article was published in the following Dove Press journal:

Therapeutics and Clinical Risk Management

\author{
Li-Fen Yang',* \\ Liang-Ming Cail,* \\ Ming $\mathrm{Li}^{2, *}$ \\ Jin-Tao Liu $^{3}$ \\ Zhao-Ni Wang' \\ Wei-Hao Wang ${ }^{4}$ \\ Qin-tai Yang ${ }^{4}$ \\ Zhuang-Gui Chen ${ }^{1,5}$ \\ 'Department of Pediatrics Intensive \\ Care Unit and Respiratory \\ Medicine, The Third Affiliated \\ Hospital of Sun Yat-sen University, \\ Guangzhou, People's Republic of \\ China; ${ }^{2}$ Department of Pulmonary \\ Diseases, The First Affiliated \\ Hospital of Sun Yat-Sen University, \\ Guangzhou, People's Republic of \\ China; ${ }^{3}$ Department of Population \\ and Quantitative Health Science, \\ Case Western Reserve University, \\ Cleveland, OH, USA; ${ }^{4}$ Department of \\ Otorhinolaryngology-Head and Neck \\ Surgery, The Third Affiliated Hospital \\ of Sun Yat-sen University, Guangzhou, \\ People's Republic of China; \\ ${ }^{5}$ Institution of Respiratory Diseases \\ of Sun Yat-sen University, Guangzhou, \\ People's Republic of China \\ *These authors contributed equally \\ to this work
}

Correspondence: Zhuang-Gui Chen Department of Pediatric Intensive Care Unit and Respiratory Medicine, The Third Affiliated Hospital of Sun Yat-sen University, No 600, Tianhe Road, Guangzhou 510630, People's

Republic of China

Tel +8602085253380

Email chenzhuanggui@।26.com
Objective: To investigate the alterative spectrum and trends of aeroallergens sensitization in children with allergic rhinitis (AR) in Guangzhou, China in the past 10 years.

Participants and methods: In this retrospective study, 4,111 children with complaints of nasal hyper-reactivity who visited the Pediatric Department and/or Otolaryngology Department from January 2007 to November 2016 were enrolled. Serum specific immunoglobulin E was measured and positive detection was made in 3,328 patients, who were, therefore, diagnosed with AR. Positive rates and trends of different aeroallergens sensitization were assessed. The tendency of positive rates changing over the years, and the difference and trends in positive rate of aeroallergen sensitization that occurred in subgroups of gender, age, and season were determined and analyzed with logistic regression.

Results: The percentage of detected common aeroallergens in AR children was (from high to low) $81.07 \%, 34.44 \%, 14.72 \%, 11.81 \%, 6.04 \%$, and $3.70 \%$ for house dust mites (HDMs), cat-dog dander, cockroach, mold mixture, tree pollen mixture, and herb pollen mixture, respectively. An ascending trend of aeroallergens sensitization or AR (odds ratio [OR] $=1.116,95 \%$ CI: $1.086-1.146$ ) was found. Interestingly, an increasing trend of cat-dog dander and mold sensitization was found in AR children ( $\mathrm{OR}=1.164,95 \% \mathrm{CI}: 1.133-1.196$; OR $=1.169,95 \% \mathrm{CI}: 1.120-1.223)$ in this retrospective study, while HDMs sensitization held a steady trend (OR $=0.983,95 \%$ CI: 0.961-1.007). Conclusion: In the increasing trend of aeroallergens sensitization or AR, HDMs sensitization still held the majority. But emphasis should be made on pet allergy for young children with AR in the context of ascending trend of sensitization to cat-dog dander.

Keywords: allergic rhinitis, aeroallergen, children, sIgE

\section{Introduction}

Allergic rhinitis (AR) is an immunoglobulin E (IgE)-mediated type I allergic disorder with characteristic nasal hyper-reactivity symptoms including nasal pruritus, sneezing, and airflow obstruction. With an increasing prevalence over the last few decades shown by previous studies, AR, not surprisingly, has become a well-recognized allergic disease in children. ${ }^{1,2}$ Detection of aeroallergens sensitization is of great importance in diagnosing AR and optimizing the care of AR patients and management of this disorder. ${ }^{2,3}$

However, the spectrum of aeroallergens sensitization in different regions was diverse and plenty of factors affected it. In southern China, the most common allergen of asthma and/or AR was house dust mites (HDMs) because of the warm and humid climate, whereas pollen made up the majority in northern China. ${ }^{4}$ Furthermore, the sensitization spectrum also varied by the change of economic development, environmental conditions, and modern lifestyle..$^{5-7}$ Located in the eastern coastal areas 
of China, Guangzhou underwent a process of industrialization and urbanization in recent years. The rapid economic growth and constant changes of lifestyle had not only led to an increased incidence of AR but also varied the distribution of allergens, especially in urban area. ${ }^{8}$

For general population in Guangzhou, the aeroallergensensitized spectrum was found altered in the past 10 years in our previous study. ${ }^{9}$ However, reports of dynamic changes in the constitution of aeroallergens in children with AR in southern China were still limited.

Thus, we aimed to retrospectively analyze the changing spectrum of aeroallergens sensitization in Guangzhou in the past decade, and provide evidence for promoting effective diagnosis and therapy in children with AR.

\section{Participants and methods Study design and participants}

All cases who visited the Pediatric Department and/or Otolaryngology Department of the Third Affiliated Hospital of Sun Yat-sen University with complaints of nasal hyper-reactivity from January 2007 to November 2016 were reviewed. For children with multiple visits at the hospital or multiple tests of specific immunoglobulin E (sIgE), only results of the first test would be counted. Patients had to be aged from 2 months to 18 years. Further analysis would be conducted if patients fulfilled the diagnosis criteria of the guideline of pediatric AR: 1) present to the doctor with symptoms of nasal hyper-reactivity such as sneezing and a runny, itchy, or blocked nose; 2) positive detection of serum sIgE of aeroallergens. ${ }^{10}$ Patients with one of the following conditions would be excluded: 1) a history of other allergic diseases such as asthma or atopic dermatitis; 2) not a local resident of Guangzhou (not a permanent resident of Guangzhou or living in Guangzhou less than a year). These following measurable clinical items were included: 1) age at the detection of serum sIgE; 2) gender; 3 ) the result of serum sIgE tests; 4) season at the detection of serum sIgE.

The patients were divided into four age groups according to age at the detection of sIgE: 1) infancy and toddler, 0-3 years; 2) preschool, 4-6 years; 3) school-age, 7-12 years; and 4) adolescence, 13-18 years. Meanwhile, the season group was evaluated by months in the detection of sIgE, and was classified as 1) spring, from March to May; 2) summer, from June to August; 3) autumn, from September to November; 4) winter, from December to February.

\section{Aeroallergens detection}

Three milliliters of peripheral blood was collected from each patient. sIgE of six common aeroallergens was detected using the Allergy Screen System (Mediwiss Analytic GmbH, Moers, Germany). These aeroallergens include HDMs, cat-dog dander, cockroach, mold mixture (Penicillium notatum, branch spore mildew, Aspergillus fumigatus, and Alternaria), tree pollen mixture (combination of cypress, elm, phoenix tree, willow, and cottonwood), and herb pollen mixture (combination of short ragweed, mugwort, Humulus scandens, and pigweed). All participants received testing to this allergens panel. The serum sIgE level was expressed as a concentration of international units per milliliter $(\mathrm{IU} / \mathrm{mL})$. The results of sIgE were graded from Class 0 to 6 according to the concentration gradient provided by Allergy Screen System: Class 0, 0-0.35 IU/mL; Class 1, 0.36-0.75 IU/mL; Class 2, 0.76-3.5 IU/mL; Class 3, 3.6-17.5 IU/mL; Class 4, 17.6-50 IU/mL; Class 5, 51-100 IU/mL; Class 6, higher than $100 \mathrm{IU} / \mathrm{mL}$. An sIgE measure of $0.36 \mathrm{IU} / \mathrm{mL}$ or more was defined as positive detection.

\section{Statistical analysis}

Data were analyzed using IBM-SPSS Statistics (version 20.0; IBM, NY, USA) and SAS (version 9.4; SAS Inc., Cary, NC, USA). Continuous data were presented as the mean and SD while categorical data were presented as percentages. Significant difference between the positive rates of different subgroups (gender, season, and age) was determined by Pearson's chi-square test. Pearson's correlation analysis was used to analyze whether correlation existed between the positive rates and years. Logistic regression was used to analyze how the positive rate of aeroallergen sensitization changed as age increased and the tendency of positive rates changing over the years. Odds ratio (OR) with 95\% CI was given. For all analyses, differences were considered statistically significant when $P$-values were $<0.05$.

\section{Ethical approval}

This study was approved by the Ethics Committee of the Third Affiliated Hospital of Sun Yat-sen University. The ethics committee waived the need for informed consent as only de-identified patient data were used, and no human intervention was involved.

\section{Results}

\section{Overall positive rate of aeroallergen sensitization in children with nasal hyper-reactivity}

A total of 4,111 patients (2,669 male and 1,442 female) with a symptom of nasal hyper-reactivity were enrolled in this present study. Among them, positive detection of serum 
sIgE was found in 3,328 (80.95\%) patients (2,205 male and 1,123 female), who were, therefore, diagnosed as AR.

\section{Positive rate of aeroallergens sensitization in AR children and its changes}

In patients with AR, six common aeroallergens were detected and the positive rates are shown in Table 1 . The prevalence was the highest for HDMs (81.07\%), followed by cat-dog dander (34.44\%) and cockroach (14.72\%), while the lowest was for herb pollen mixture (3.70\%) (Table 1; Figure 1A).

\section{Changes in the positive rate of aeroallergens sensitization over the past 10 years}

To further assess the impact of increasing years on the spectrum of aeroallergens sensitization in children diagnosed with AR, we analyzed the positive rate of each aeroallergen in the past 10 years (Table 1). Positive correlation was found between the year and the overall aeroallergens sensitization $(P<0.01)$ and an ascending trend was seen in the last 10 years ( $\mathrm{OR}=1.116$; Figure 1B). With $95 \% \mathrm{CI}$, the odds of aeroallergens positive rate or AR changed by an amount between 1.09 and 1.15 times for every 1 year increase, after controlling for other covariates $(P<0.001)$. In AR children, positive rate of HDM sensitization decreased from $90.91 \%$ in 2007 to $78.85 \%$ in 2016 and was negatively correlated with year $(P<0.01)$. But logistic regression after controlling for age, gender, and season as covariates showed that OR of the positive rate of HDMs was 0.983 ( $95 \% \mathrm{CI}$ : $0.961-1.007, P=0.163$; Figure 1C). However, sensitization of cat-dog dander (from
$29.54 \%$ in 2007 to $44.67 \%$ in 2016 ) and mold (from $4.63 \%$ in 2007 to $12.35 \%$ in 2016) showed positive correlations with years (both $P<0.05$ ) and ascending trend was observed (OR $=1.164,95 \%$ CI: $1.133-1.196, P<0.001$; OR $=1.169$, 95\% CI: $1.120-1.223, P<0.001$; Figure 1D and E). Cockroach, tree, and herb pollen were not relevant to year.

\section{Positive rates of different aeroallergens in AR children grouped by gender, season, and age}

In the gender subgroup, male had more chance of being sensitized to aeroallergens than female $(82.61 \%$ vs $77.88 \%$, $P<0.001)$. Difference of sensitization distribution in AR children grouped by gender can be found in three allergens, HDM, herb pollen, and mold. Male in our study had a higher proportion of sensitization to HDM $(82.54 \%$ vs $78.18 \%$, $P=0.002)$ and herb pollen ( $4.31 \%$ vs $2.49 \%, P=0.009)$ than female while a lower proportion to mold mixture $(10.98 \%$ vs $13.45 \%, P=0.037$ ) (Figure $2 \mathrm{~A}$ ).

Numbers and positive rates of patients with AR in different seasons were as follows: 521 in spring (84.99\%), 1,280 in summer (92.49\%), 467 in autumn (88.11\%), and 497 in winter $(84.81 \%)$. When comparisons by seasons were performed in patients with the same positive aeroallergens, we found that positive rate of cockroach was lower in spring ( $P=0.01)$ while the positive rate of HDM was the highest in summer $(P<0.001)$. No significant difference was observed in other groups (Figure 2B).

Numbers of patients with AR in different age groups were as follows: 482 in infancy and toddler, 905 in preschool,

Table I Positive rate (\%) of aeroallergens sensitization in children with AR by year

\begin{tabular}{|c|c|c|c|c|c|c|c|}
\hline Year & Overall & HDMs (\%) & $\begin{array}{l}\text { Cat-dog } \\
\text { dander (\%) }\end{array}$ & Cockroach (\%) & Mold (\%) & $\begin{array}{l}\text { Herb } \\
\text { pollen (\%) }\end{array}$ & $\begin{array}{l}\text { Tree } \\
\text { pollen (\%) }\end{array}$ \\
\hline Total & 3,328 & $2,698(81.07)$ & $\mathrm{I}, \mathrm{I} 46$ (I4.72) & $490(14.72)$ & $393(11.81)$ & $123(3.70)$ & $201(6.04)$ \\
\hline 2007 & 281 & 258 (9I.8I) & 83 (29.54) & 48 (I7.08) & $13(4.63)$ & $10(3.56)$ & 21 (7.47) \\
\hline 2008 & 142 & 132 (92.96) & $24(16.90)$ & 18 (12.68) & $8(5.63)$ & 7 (4.93) & $3(2.11)$ \\
\hline 2009 & 153 & $129(84.3 \mathrm{I})$ & 35 (22.88) & 23 (I5.03) & $14(9.15)$ & $13(8.50)$ & $6(3.92)$ \\
\hline 2010 & 123 & II 2 (9I.06) & $23(18.70)$ & $35(28.46)$ & $8(6.50)$ & II (8.94) & $6(4.88)$ \\
\hline 2011 & 178 & I45 (8I.46) & $38(21.35)$ & $33(18.54)$ & $22(12.36)$ & $9(5.06)$ & $3(1.69)$ \\
\hline 2012 & 212 & $182(85.85)$ & $68(32.08)$ & 38 (17.92) & $16(7.55)$ & $17(8.02)$ & $13(6.13)$ \\
\hline 2013 & 493 & $386(78.30)$ & $188(38.13)$ & $7 \mid(14.40)$ & 64 (12.98) & $12(2.43)$ & $25(5.7 \mathrm{I})$ \\
\hline 2014 & 576 & 472 (81.94) & I47 (25.52) & 75 (13.02) & $54(9.38)$ & $9(1.56)$ & $26(4.5 \mathrm{I})$ \\
\hline 2015 & 579 & $4 I 6$ (7I.85) & $276(47.67)$ & $86(14.85)$ & I2I (20.90) & $16(2.76)$ & $33(5.70)$ \\
\hline 2016 & 591 & $466(78.85)$ & $264(44.67)$ & $63(10.66)$ & $73(12.35)$ & $19(3.21)$ & $65(11.00)$ \\
\hline $\begin{array}{l}\text { Pearson's correlation } \\
\text { coefficient }\end{array}$ & $0.867 * *$ & $-0.84 \mathrm{It \dagger}^{\mathrm{t}}$ & $0.740^{\ddagger}$ & -0.0355 & $-0.737^{\S}$ & -0.473 & 0.446 \\
\hline
\end{tabular}

Notes: **Refers to the overall positive rate of aeroallergens sensitization in children with $A R$ was found correlated with year and $P$-value was $<0.0 \mathrm{I}$ but $\geq 0.00 \mathrm{I}$. ${ }^{\dagger+R e f e r s ~ t o ~}$ the positive rate of HDM sensitization in children with AR was found correlated with year and $P$-value was $<0.01$ but $\geq 0.001$. ${ }^{*}$ Refers to the positive rate of cat-dog dander sensitization in children with $A R$ was found correlated with year and $P$-value was $<0.05$ but $\geq 0.01$. ${ }^{\S}$ Refers to the positive rate of mold sensitization in children with AR was found correlated with year and $P$-value was $<0.05$ but $\geq 0.01$.

Abbreviations: AR, allergic rhinitis; HDM, house dust mite. 


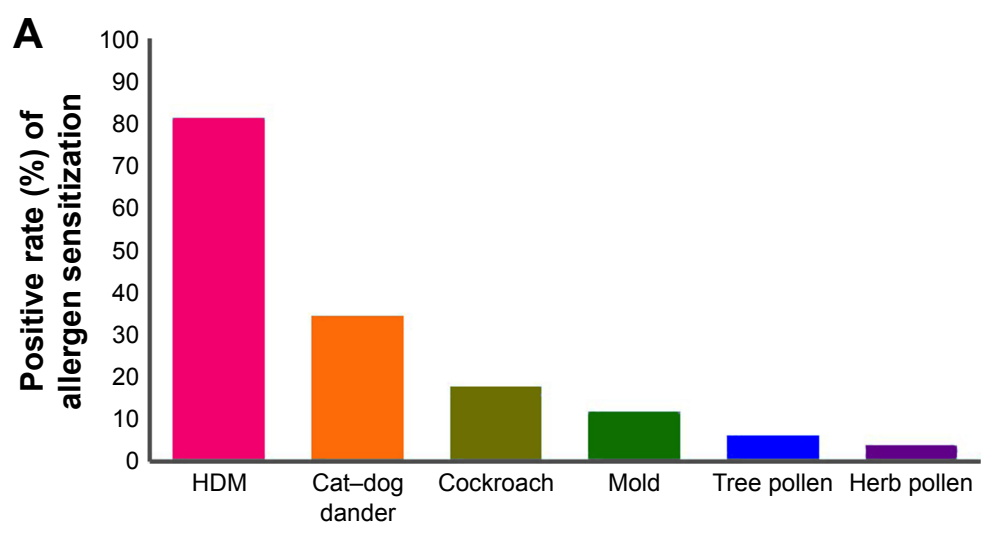

Aeroallergens
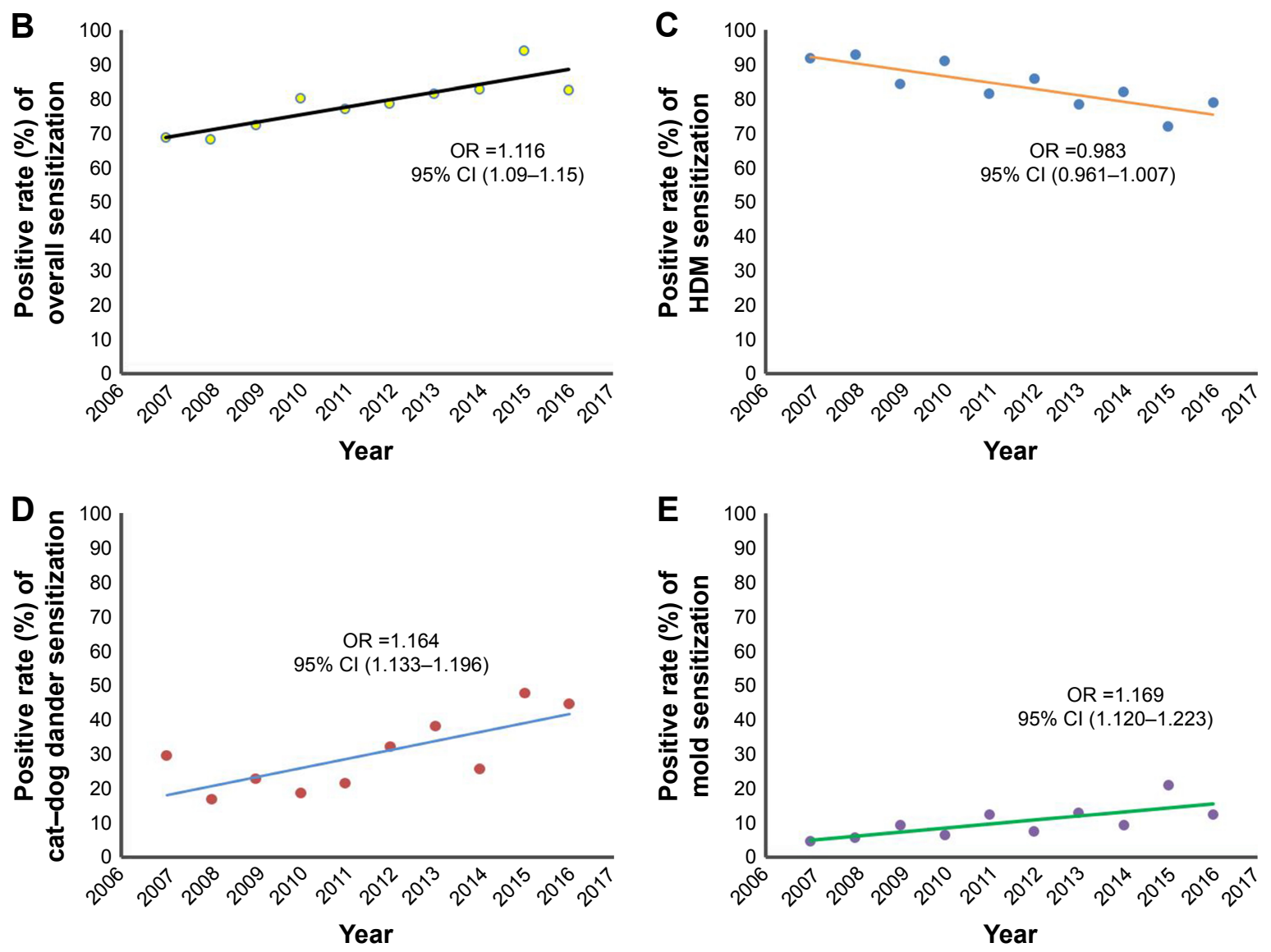

Figure I Positive rate (\%) of the slgE of six aeroallergens in children with AR (A). The ascending trend of the positive rate (\%) of being allergic to at least one aeroallergen in patients from 2007 to 2016 (B). Change of the positive rates of HDM (C), cat-dog dander (D), and mold (E) in AR children during the past I0 years.

Abbreviations: AR, allergic rhinitis; HDM, house dust mite; OR, odds ratio; slgE, specific immunoglobulin $\mathrm{E}$.

1,458 in school-age, and 683 in adolescent. The positive rates of sensitization to various aeroallergens for different age groups are shown in Table 2. The highest prevalence of HDM sensitization in AR children was in school-age $(86.80 \%)$ while sensitization to cat-dog dander was higher in infancy and toddler $(40.46 \%)$ than in other age groups. Significant differences could be observed in HDM and cockroach sensitization when age increased.

\section{Discussion}

In the present study, we revealed some interesting changes of the aeroallergens sensitization spectrum in children with AR. The prevalence of aeroallergens sensitization was steadily increasing in the past decade in China, as reported by others. ${ }^{11,12}$ HDM was targeted as the most common aeroallergen for either adult or children AR in southern China. ${ }^{13-15}$ Located in the subtropical zone and having a hot and humid 

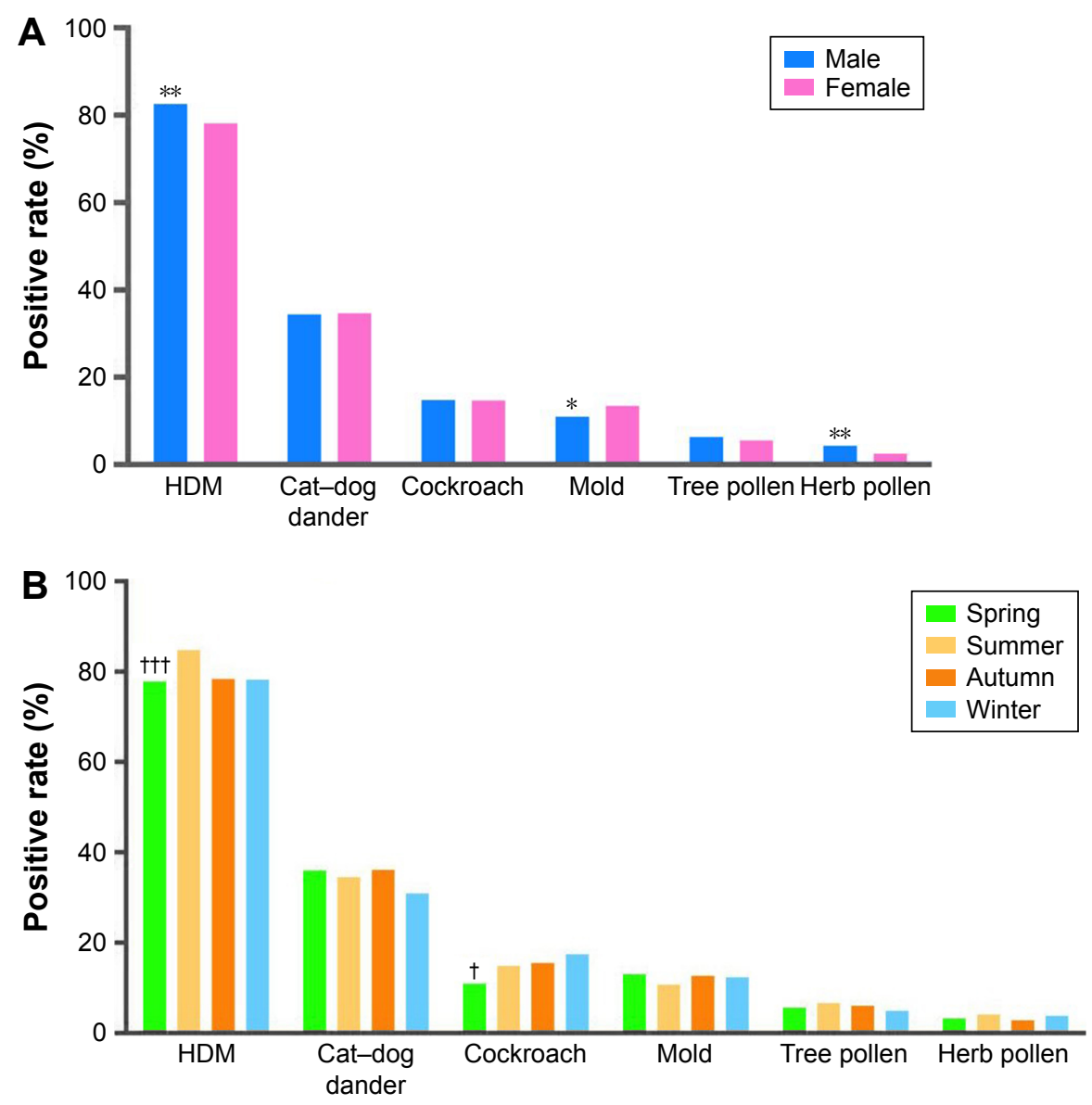

Figure 2 Positive rate (\%) of the slgE of each aeroallergen in patients with AR by gender $(\mathbf{A})$ and season (B).

Notes: $*$ and $* *$ indicate significant differences of the positive rates of aeroallergens sensitization in children with AR could be found between male and female. $*$ indicates $P$-value was $<0.05$ but $\geq 0.01$. ** indicates $P$-value was $<0.01$ but $\geq 0.001$. ${ }^{\dagger}$ and ${ }^{t+t}$ indicate significant differences of the positive rates of aeroallergens sensitization in children with AR could be found between different seasons. ${ }^{\dagger}$ indicates $P$-value was $<0.05$ but $\geq 0.01$. ${ }^{t+t}$ indicates $P$-value was $<0.00 \mathrm{I}$.

Abbreviations: AR, allergic rhinitis; HDM, house dust mite; slgE, specific immunoglobulin $E$.

weather, Guangzhou has provided a perfect environment for HDMs to raise and reproduce, as high humidity and ambient temperatures were reported to be optimal conditions for HDM propagation. ${ }^{16}$ Concentration of HDMs met its peak in August indoors. ${ }^{17}$ Spending more time indoors in summer combining with poor ventilation also largely increased exposure to HDMs. ${ }^{18}$ This explained why severe sensitization to HDMs could be found in summer by us. At the same time, the exacerbating air pollution caused by automobile exhaust also contributes to the high detection of HDMs. ${ }^{19}$ In the context of high prevalence of HDMs sensitization, specific immunotherapy (SIT) of HDMs should be strongly considered by pediatricians. SIT had been proved to be effective and listed as the first-line treatment by guidelines for pediatric AR.,10

Although a slightly decreasing trend was revealed by us in the 10-year observation, HDMs sensitization still held the majority in AR children. In our study, we found that the prevalence of sensitizing to cat-dog dander significantly increased. In this regard, we presumed that the descending proportion of HDMs sensitization may be associated with the ascending trend of sensitization to other allergens, such as cat-dog dander. More data would be needed and a long-term prospective study would be of great importance in revealing reasons behind changes.

The significantly increased trend of cat-dog dander suggested that pets' aeroallergens might be a critical factor for prevention and treatment of allergic diseases in the urbanization regions in the future. The reason to that might attribute to the rapid development of economy and changes of lifestyle in recent years, which leads to the process of industrialization and urbanization, especially in regions of southern China. Currently, the proportion of families that chose to keep cats and dogs as pets also raised, largely increasing the risk of exposure to cat-dog dander allergens. ${ }^{9}$ Having household pets at home was found to be a risk factor for inducing airway diseases..$^{20}$ The more contacts one person has with pet allergens, the more possibility he or she develops a symptom of airway hyper-reactivity. ${ }^{21}$ However, the European PASTURE 
Table 2 Positive rate (\%) of aeroallergens sensitization in children with AR by age group

\begin{tabular}{|c|c|c|c|c|c|c|}
\hline \multirow[t]{2}{*}{ Aeroallergen } & \multirow[t]{2}{*}{ Age group } & \multirow{2}{*}{$\begin{array}{l}\text { Number } \\
\text { of positive } \\
\text { detection }\end{array}$} & \multirow{2}{*}{$\begin{array}{l}\text { Positive } \\
\text { rate (\%) }\end{array}$} & \multicolumn{3}{|l|}{ OR $(95 \% \mathrm{CI})$} \\
\hline & & & & Preschool vs & School-age vs & Adolescent vs \\
\hline \multirow[t]{4}{*}{ HDM } & Infancy and toddler & 292 & 60.58 & $2.55(2.11-3.08)^{* * * *}$ & $5.17(4.25-6.29)^{t+t}$ & $5.48(4.34-6.93)^{\ddagger \ddagger}$ \\
\hline & Preschool & 726 & 80.22 & & $2.03(I .7 I-2.4 I)^{\S \S \S}$ & $2.15(1.74-2.67)^{\# \#}$ \\
\hline & School-age & 1,092 & 86.80 & & & 1.06 (0.86-I.32) \\
\hline & Adolescent & 588 & 86.09 & & & \\
\hline Cat-dog & Infancy and toddler & 195 & 40.46 & $1.02(0.82-1.26)$ & $1.10(0.89-1.35)$ & $0.99(0.78-1.25)$ \\
\hline \multirow[t]{3}{*}{ dander } & Preschool & 318 & 35.14 & & $1.08(0.9|-| .29)$ & $0.97(0.70-1.20)$ \\
\hline & School-age & 413 & 32.83 & & & $0.90(0.74-1.10)$ \\
\hline & Adolescent & 220 & 32.21 & & & \\
\hline \multirow[t]{4}{*}{ Cockroach } & Infancy and toddler & 51 & 10.58 & $1.54(1.08-2.23)^{*}$ & $2.76(1.99-3.92)^{t+\dagger}$ & $2.89(2.01-4.22)^{\ddagger \ddagger \ddagger}$ \\
\hline & Preschool & 110 & 12.15 & & $1.79(I .40-2.31)^{\S \S \S}$ & I.87 (I.40-2.5I I \#\# \\
\hline & School-age & 213 & 16.93 & & & $\mathrm{I} .05(0.8 \mathrm{I}-\mathrm{I} .34)$ \\
\hline & Adolescent & 116 & 16.98 & & & \\
\hline \multirow[t]{4}{*}{ Mold mixture } & Infancy and toddler & 52 & 10.78 & $1.37(0.97-1.96)$ & $1.59(1.14-2.24)^{\dagger \dagger}$ & $1.46(1.01-2.13)^{\ddagger}$ \\
\hline & Preschool & 110 & 12.15 & & I.58 (0.89-I.5I) & $1.06(0.78-1.45)$ \\
\hline & School-age & 150 & 11.92 & & & $0.92(0.68-1.22)$ \\
\hline & Adolescent & 81 & II.86 & & & \\
\hline Tree pollen & Infancy and toddler & 24 & 4.98 & I.44 (0.78-2.89) & $1.87(1.03-3.65)^{\dagger}$ & $2.19(1.12-4.48)^{\ddagger}$ \\
\hline \multirow[t]{3}{*}{ mixture } & Preschool & 44 & 4.86 & & $1.29(0.8 I-2.10)$ & $1.52(0.88-2.62)$ \\
\hline & School-age & 90 & 7.15 & & & $1.17(0.7 \mathrm{I}-1.89)$ \\
\hline & Adolescent & 43 & 6.30 & & & \\
\hline Herb pollen & Infancy and toddler & 17 & 3.53 & $1.25(0.75-2.14)$ & $2.07(1.31-3.43)^{\dagger \dagger}$ & $1.73(1.02-2.99)^{\ddagger}$ \\
\hline \multirow[t]{3}{*}{ mixture } & Preschool & 32 & 3.54 & & $1.66(1.15-2.42)^{\S \S}$ & $1.38(0.89-2.14)$ \\
\hline & School-age & 47 & 3.74 & & & $0.83(0.56-1.20)$ \\
\hline & Adolescent & 27 & 3.95 & & & \\
\hline
\end{tabular}

Notes: * and *** indicates significant differences of the positive rates of aeroallergens sensitization in children with AR could be found between Preschool and Infancy and toddler. $*$ indicates $P$-value was $<0.05$ but $\geq 0.01$. $* * *$ indicates $P$-value was $<0.001$. ${ }^{t, t \dagger}$ and ${ }^{{ }^{t+t}}$ indicates significant differences of the positive rates of aeroallergens sensitization in children with AR could be found between School-age and Infancy and toddler. ${ }^{\dagger}$ indicates $P$-value was $<0.05$ but $\geq 0.0 \mathrm{I}$. ${ }^{\dagger+}$ indicates $P$-value was $<0.0 \mathrm{I}$ but $\geq 0.00 \mathrm{I}$.

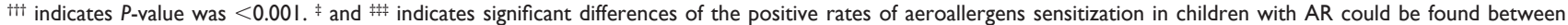
Adolescent and Infancy and toddler. ${ }^{\ddagger}$ indicates $P$-value was $<0.05$ but $\geq 0.01$. ${ }^{\ddagger \neq}$ indicates $P$-value was $<0.001 .{ }^{\S \S}$ and ${ }^{\$ \$ \S}$ indicates significant differences of the positive rates of aeroallergens sensitization in children with AR could be found between School-age and Preschool. ${ }^{\S \S}$ meant $P$-value was $<0.0$ I but $\geq 0.00$ I. ${ }^{\$ \S \S}$ indicates $P$-value was $<0.00$ I. indicates significant differences of the positive rates of aeroallergens sensitization in children with AR could be found between Adolescent and Preschool. $P$-value was $<0.001$. The positive rates within this table equaled the numbers of patients of a certain age group sensitizing to one specific allergen divided to the numbers of patients of this age group.

Abbreviations: AR, allergic rhinitis; HDM, house dust mite; OR, odds ratio.

project (Protection against Allergy-Study in Rural Environments) has suggested that pet keeping, especially cats and dogs, in early stage of one's life was inversely related to the development of allergic symptoms. ${ }^{22}$ These contradictory findings suggested that pet allergens sensitization might have a complicated mechanism and affected by multiple factors.

We also found that mold has an ascending trend in the past 10 years. Both indoor and outdoor mold allergens were included in our detection so it was hard to attribute the increasing mold sensitization to one single activity. But decreased outdoor activities due to urbanization in Guangzhou might have a say in it. Change of living environment and modern lifestyle was evident to be risk factors of AR by another research focusing on general population of Guangzhou. ${ }^{8}$ A cross-sectional population-based study conducted by previous researchers had revealed that exposure to mold at home could contribute to current AR. ${ }^{23}$ Mold exposure in infancy also increased the risk of current AR. ${ }^{24}$ Physicians should attach importance to mold sensitization for the future risk of developing AR.

The total positive rate of sIgE was significantly higher in men than in women. Arbes et al had found that test responses to allergen were more likely to be positive in men. ${ }^{25}$ Male gender was found to be a risk factor for allergen sensitizations in a cross-sectional survey performed in 6,304 patients with asthma and/or rhinitis in China. ${ }^{26} \mathrm{~A}$ plausible explanation was that the total IgE plasma levels were higher in men with $\mathrm{AR}$, as reported by others. ${ }^{27}$

In addition, age was also found to affect the distribution of allergens sensitization. Boulet et al had suggested that the sensitization of all allergens tended to increase and reached its highest degree in young adults. ${ }^{28}$ Sensitization to other allergens in our study except for HDMs and cockroach showed no significant difference as age increased. The reason 
to that remained unclear. More cases from different medical centers were needed in our future study to find out the relationship between age and the prevalence of aeroallergen sensitization.

Due to the imperfect family doctor system, patients who developed symptoms tended to visit a first-class hospital for getting more accurate diagnosis and better therapeutic options in China. Thus, the patients enrolled in our study had a relatively high representation of the general population with nasal hyper-reactivity in Guangzhou. Yet, based on the fact that our study was a single-center study, more caution should be paid before applying our findings to children with $\mathrm{AR}$ in other regions. Furthermore, as a retrospective study focusing mostly on the positive rates of serum sIgE, the degree of severity of the patients' disease and their exposure to allergen were not considered in this study. To compensate these limitations, a multicenter study accompanied with multivariate analysis was needed to get more comprehensive information.

\section{Conclusion}

In the increasing trend of aeroallergens sensitization or AR, HDMs sensitization still held the majority. But emphasis should be made on pet allergy for young children with $\mathrm{AR}$ in the context of ascending trend of sensitization to cat-dog dander.

\section{Acknowledgments}

We appreciate Dr Yan Yan and Pei-Ying Feng for proofreading of manuscript. This study was supported by National Natural Science Foundation of China (Number 81470219); Science and Technology Projects Foundation of Guangdong Province (Number 2014A020212120).

\section{Disclosure}

The authors report no conflicts of interest in this work.

\section{References}

1. Barr JG, Al-Reefy H, Fox AT, Hopkins C. Allergic rhinitis in children. BMJ. 2014;349:g4153.

2. Seidman MD, Gurgel RK, Lin SY, et al. Clinical practice guideline: allergic rhinitis. Otolaryngol Head Neck Surg. 2015;152(1 Suppl): S1-S43.

3. Bousquet J, Bachert C, Canonica GW, et al; Extended Global Allergy and Asthma European Network, World Allergy Organization and Allergic Rhinitis and its Impact on Asthma Study Group. Unmet needs in severe chronic upper airway disease (SCUAD). J Allergy Clin Immunol. 2009; 124(3):428-433.

4. Li J, Sun B, Huang Y, et al; China Alliance of Research on Respiratory Allergic Disease. A multicentre study assessing the prevalence of sensitizations in patients with asthma and/or rhinitis in China. Allergy. 2009;64(7):1083-1092.
5. Van Bever HP, Lee BW, Shek LP. Viewpoint: the future of research in pediatric allergy: what should the focus be? Pediatr Allergy Immunol. 2012;23(1):5-10.

6. Storkey J, Stratonovitch P, Chapman DS, Vidotto F, Semenov MA. A process-based approach to predicting the effect of climate change on the distribution of an invasive allergenic plant in Europe. PLoS One. 2014;9(2):e88156.

7. Baumann LM, Romero KM, Robinson CL, et al; PURA Study Investigators. Prevalence and risk factors for allergic rhinitis in two resourcelimited settings in Peru with disparate degrees of urbanization. Clin Exp Allergy. 2015;45(1):192-199.

8. Li CW, Chen DH, Zhong JT, et al. Epidemiological characterization and risk factors of allergic rhinitis in the general population in Guangzhou City in china. PLoS One. 2014;9(12):e114950.

9. Wang W, Huang X, Chen Z, et al. Prevalence and trends of sensitisation to aeroallergens in patients with allergic rhinitis in Guangzhou, China: a 10-year retrospective study. BMJ Open. 2016;6(5):e11085.

10. Subspecialty Group of Rhinology, Editorial Board of Chinese Journal of Otorhinolaryngology Head and Neck Surgery; Subspecialty Group of Rhinology and Pediatrics, Society of Otorhinolaryngology Head and Neck Surgery, Chinese Medical Association; Editorial Board of Chinese Journal of Pediatrics. 儿童变应性鼻炎诊断和治疗指 南（2010年, 重庆） [Guidelines for diagnosis and treatment of pediatric allergic rhinitis (2010, Chongqing)]. Chin J Otorhinol Head Neck Surg. 2011;46(1):7-8. Chinese.

11. Zhang Y, Zhang L. Prevalence of allergic rhinitis in china. Allergy Asthma Immunol Res. 2014;6(2):105-113.

12. Shen J, Ke X, Hong S, et al. Epidemiological features of allergic rhinitis in four major cities in Western China. J Huazhong Univ Sci Technolog Med Sci. 2011;31(4):433-440.

13. Ding J, Zhang J, Xu F, Xu Y, Zhu H. 上海地区890例变应性鼻炎患 者吸入变应原谱分析 [Analyzing of the inhaled allergens profiles of 890 allergic rhinitis patients]. Lin Chung Er Bi Yan Hou Tou Jing Wai Ke Za Zhi. 2012;26(4):164-166. Chinese.

14. Huang F, Zhao Y, He J, et al. 荆门地区变应性鼻炎患者吸入变应原 谱分析 [Analyzing of the inhaled allergens profiles of allergic rhinitis patients in district of Jingmen]. Lin Chung Er Bi Yan Hou Tou Jing Wai Ke Za Zhi. 2010;24(8):341-343. Chinese.

15. Kong WJ, Chen JJ, Zheng ZY, Shi QM, Zhou Y. Prevalence of allergic rhinitis in 3-6-year-old children in Wuhan of China. Clin Exp Allergy. 2009;39(6):869-874.

16. Zhang $\mathrm{C}$, Li J, Lai X, et al. House dust mite and storage mite IgE reactivity in allergic patients from Guangzhou, China. Asian Pac J Allergy Immunol. 2012;30(4):294-300.

17. Zhang C, Gjesing B, Lai X, Li J, Spangfort MD, Zhong N. Indoor allergen levels in Guangzhou city, southern China. Allergy. 2011;66(2): 186-191.

18. Andiappan AK, Puan KJ, Lee B, et al. Allergic airway diseases in a tropical urban environment are driven by dominant monospecific sensitization against house dust mites. Allergy. 2014;69(4): 501-509.

19. Zhong J, Liu D, Huang Z. Analysis of skin prick test about aeroallergens on 2136 children with allergic rhinitis in Guangzhou area. Chin Arch Otolaryngol Head Neck Surg. 2014;21(9):481-485.

20. Dong GH, Ma YN, Ding HL, et al. Pets keeping in home, parental atopy, asthma, and asthma-related symptoms in 12,910 elementary school children from northeast China. Indoor Air. 2009;19(2):166-173.

21. Madhurantakam C, Nilsson OB, Uchtenhagen $\mathrm{H}$, et al. Crystal structure of the dog lipocalin allergen Can $\mathrm{f} 2$ : implications for cross-reactivity to the cat allergen Fel d 4. J Mol Biol. 2010;401(1):68-83.

22. von Mutius E, Schmid S; PASTURE Study Group. The PASTURE project: EU support for the improvement of knowledge about risk factors and preventive factors for atopy in Europe. Allergy. 2006;61(4): 407-413.

23. Azalim S, Camargos P, Alves AL, Senna MI, Sakurai E, Schwabe KW. Exposure to environmental factors and relationship to allergic rhinitis and/or asthma. Ann Agric Environ Med. 2014;21(1):59-63. 
24. Yang SI, Lee E, Jung YH, et al. Effect of antibiotic use and mold exposure in infancy on allergic rhinitis in susceptible adolescents. Ann Allergy Asthma Immunol. 2014;113(2):160.e1-165.e1.

25. Arbes SJ, Gergen PJ, Elliott L, Zeldin DC. Prevalences of positive skin test responses to 10 common allergens in the US population: results from the third National Health and Nutrition Examination Survey. J Allergy Clin Immunol. 2005;116(2):377-383.

26. Li J, Huang Y, Lin X, et al; China Alliance of Research on Respiratory Allergic Disease (CARRAD). Factors associated with allergen sensitizations in patients with asthma and/or rhinitis in China. Am J Rhinol Allergy. 2012;26(2):85-91.
27. Paula Couto T, Falsarella N, Mattos Cde C, Mattos LC. Total IgE plasma levels vary according to gender and age in Brazilian patients with allergic rhinitis. Clinics (Sao Paulo). 2014;69(11):740-744.

28. Boulet LP, Turcotte H, Laprise C, et al. Comparative degree and type of sensitization to common indoor and outdoor allergens in subjects with allergic rhinitis and/or asthma. Clin Exp Allergy. 1997;27(1): 52-59.

\section{Publish your work in this journal}

Therapeutics and Clinical Risk Management is an international, peerreviewed journal of clinical therapeutics and risk management, focusing on concise rapid reporting of clinical studies in all therapeutic areas, outcomes, safety, and programs for the effective, safe, and sustained use of medicines. This journal is indexed on PubMed Central, CAS,
EMBase, Scopus and the Elsevier Bibliographic databases. The manuscript management system is completely online and includes a very quick and fair peer-review system, which is all easy to use. Visit http://www.dovepress.com/testimonials.php to read real quotes from published authors.

Submit your manuscript here: http://www.dovepress.com/therapeutics-and-clinical-risk-management-journal 